\title{
Prueba de desaturación y titulación de oxígeno suplementario. Recomendaciones y procedimientos
}

\author{
Oxygen desaturation and supplemental oxygen \\ titration tests. A proposal of standardization
}

\author{
Claudia Vargas-Domínguez,* Roberto Mejía-Alfaro,* Ramiro Martínez-Andrade,* \\ Mónica Silva-Cerón, ${ }^{*}$ Juan Carlos Vázquez-García, ${ }^{*}$ Luis Torre-Bouscoulet*
}

*Departamento de Fisiología Respiratoria; Instituto Nacional de Enfermedades Respiratorias; Ciudad de México.

\begin{abstract}
RESUMEN. La prueba de desaturación de oxígeno y la prueba de titulación de oxígeno suplementario son realizadas con fines diagnósticos, pronósticos y terapéuticos. Con el propósito de incrementar la precisión y la exactitud de los resultados, nos dimos a la tarea de optimizar el proceso técnico que se lleva a cabo durante la ejecución de las pruebas. Los diagramas de flujo que proponemos en este manuscrito facilitan la toma de decisiones durante la realización de las pruebas y recomendamos que la pertinencia de cada prueba sea evaluada a la luz del estado físico del paciente. La utilidad práctica de dichos diagramas la hemos confirmado durante las actividades cotidianas de nuestro laboratorio; sin embargo, su aplicabilidad en otros laboratorios de función pulmonar debe de ser explorada. Aunque ha sido diseñado como una estrategia de control de calidad de nuestro laboratorio, los autores creemos que este informe podría ser de utilidad para otros centros interesados en realizar estas pruebas.
\end{abstract}

Palabras clave: Estandarización, prueba de desaturación de oxígeno, titulación de oxígeno suplementario, ejercicio, función respiratoria.

\section{Abreviaturas:}

ATS: Sociedad Americana del Tórax (American Thoracic Society)

C6M: Caminata de 6 minutos

ERS: Sociedad respiratoria europea (European Respiratory Society)

LFM: Limitaciones físicas mayores

LFP: Limitaciones físicas parciales

$\mathrm{PaCO}_{2}$ : Presión parcial de bióxido de carbono en sangre arterial

$\mathrm{PaO}_{2}$ : Presión parcial de oxígeno en sangre arterial

PDO: Prueba de desaturación de oxígeno

PECP: Prueba de ejercicio cardiopulmonar

PFRs: Pruebas de función respiratoria

PTOS : Prueba de titulación de oxígeno suplementario

Correspondencia:

\section{Dr. Luis Torre-Bouscoulet}

Departamento de Fisiología Respiratoria,

Instituto Nacional de Enfermedades Respiratorias.

Tlalpan Núm. 4502, Colonia Sección XVI,

14080, Ciudad de México. Tel. y Fax: (52) (55) 56654748

Correo electrónico: luistorreb2001@yahoo.com.mx
ABSTRACT. Oxygen desaturation and supplementary oxygen titration tests are carried out for diagnostic, prognostic and therapeutic purposes. In order to enhance the precision and accuracy of the results obtained using these tests, we undertook a strategy designed to optimize the technical process performed during their execution. The flow charts presented in this manuscript facilitate the decision-making process during test performance. The authors recommend that the necessity of carrying out these tests be evaluated in relation to the physical status of each patient. The practical utility of the charts has been confirmed during the daily activities at our laboratory; however, their applicability in other pulmonary function laboratories must be explored. Though they were designed at our laboratory as a new quality control strategy, the authors believe that they may be useful for other centers that are also interested in conducting such tests.

Keywords: Standardization, oxygen desaturation test, supplemental oxygen titration, exercise, pulmonary function test.

$\mathrm{SaO}_{2}$ : Saturación de oxígeno obtenida mediante gasometría

SLF: Sin limitaciones físicas

$\mathrm{SpO}_{2}$ : Saturación de oxígeno obtenida mediante oximetría de pulso.

VMC6M: Versión modificada de la caminata de 6 minutos.

\section{INTRODUCCIÓN}

La estandarización de las pruebas de función respiratoria (PFR) hace referencia al proceso que simplifica, unifica y especifica los criterios y métodos que deben ser utilizados durante su ejecución. ${ }^{1}$ Mediante la estandarización, se minimiza la variabilidad y se logra que las pruebas sean «intercambiables»; es decir, que cualquier técnico capacitado, en cualquier sitio y con la infraestructura suficiente, realice la prueba de la misma manera obteniendo con ello resultados semejantes que podrán ser comparables entre sujetos, o bien, comparables con los resultados del mismo sujeto en relación al tiempo, generalmente en el seguimiento de enfermedad pulmonar. 
El proceso de estandarización de PFR ha sido, desde hace años, materia de debate. En el año 2005 y después de varios intentos individuales, se conjuntaron los esfuerzos de las dos organizaciones más importantes del mundo en materia de salud respiratoria (la American Thoracic Society, ATS; y la European Respiratory Society, ERS) y se publicaron los «Estándares ATS-ERS» recomendados para las PFR..$^{1-5} \mathrm{~A}$ pesar de la incuestionable relevancia del proceso de estandarización en la medición de la función pulmonar, existen algunas pruebas para las cuales los criterios y los métodos para su realización no son lo suficientemente explícitos para garantizar que ésta se realice de manera uniforme. Tal es el caso de la prueba de desaturación de oxígeno (PDO) y la prueba de titulación de oxígeno suplementario (PTOS). ${ }^{6}$

Ambas, la PDO y la PTOS, son complementarias ya que la primera evalúa el grado de desaturación de oxígeno (hipoxemia) que se presenta durante el ejercicio y la segunda evalúa el efecto del oxígeno suplementario sobre la saturación de oxígeno. El principio de la PDO radica en que en individuos sanos al realizar ejercicio, la presión parcial de oxígeno en sangre arterial $\left(\mathrm{PaO}_{2}\right)$ y la saturación de oxígeno $\left(\mathrm{SaO}_{2}\right)$ se mantienen sin cambios o se incrementan, mientras que en pacientes con enfermedades pulmonares o cardiovasculares, la oxigenación disminuye. ${ }^{7}$ Una PDO positiva tiene implicaciones fisiopatológicas, diagnósticas, terapéuticas y pronósticas. ${ }^{8-15}$

Con el propósito de incrementar la precisión (reproducibilidad) y la exactitud de los resultados obtenidos de la PDO y de la PTOS, nos dimos a la tarea de optimizar la estandarización disponible de dichas pruebas. A diferencia de lo propuesto por la ATS, ${ }^{6}$ en este documento agregamos algunos diagramas de flujo (Anexo 1) que facilitan la toma de decisiones durante la ejecución de la PDO y de la PTOS; además, proponemos que la pertinencia de cada prueba sea evaluada a la luz del estado físico del paciente ya que, de manera frecuente, son solicitadas para pacientes con impedimentos físicos mayores. Aunque se ha diseñado como una estrategia de control de calidad de nuestro laboratorio, los autores creemos que este informe podría ser de utilidad para otros Centros interesados en realizar estas pruebas.

\section{RECOMENDACIONES GENERALES}

Existen algunas recomendaciones generales que se deben tomar en consideración antes de realizar las pruebas. ${ }^{6}$ Brevemente, se debe de solicitar al paciente que el día programado para la realización de las pruebas (PDO y/o PTOS) acuda al laboratorio sin haber realizado ejercicio, sin haber fumado o exponerse a humo de tabaco de manera pasiva en la última hora y sin haber suspendido los medicamentos de uso regular. Otras recomendaciones son: remover el esmalte de uñas para minimizar el error de medición del oxímetro de pulso, acudir vistiendo ropa y calzado cómodos para ejercicio y haber desayunado algo ligero.

Antes de comenzar las pruebas, el personal técnico debe verificar que se cuente con todo el equipo necesario: oxímetro de pulso (preferentemente que evite artefactos de movimiento), estetoscopio, manómetro de presión arterial, puntas nasales para oxígeno, y disponibilidad de una toma de oxígeno, escala de Borg en formato impreso (Anexo 2), catéter arterial, jeringas, guantes, etc. Aunque la PDO y la PTOS son de bajo riesgo por tratarse de pruebas en las que el paciente realiza ejercicio submáximo, es necesario que se cuente con un «equipo de reanimación vital avanzada» (desfibrilador, cánulas orotraqueales, medicamentos antiarrítmicos, etc). Los pacientes deben de firmar un formato de autorización para realizar cualquier prueba que implique un esfuerzo físico y el personal técnico deberá cerciorarse de que no existan contraindicaciones (Anexo 3).

\section{PRUEBA DE DESATURACIÓN DE OXÍGENO}

La PDO está indicada en pacientes que cursan con síntomas respiratorios, usualmente disnea, en quienes las pruebas en reposo no permiten identificar alteraciones en el intercambio de gases. El ejemplo clásico es el paciente con alguna de las enfermedades parenquimatosas difusas quien, en etapas iniciales, manifiesta disnea sólo durante el ejercicio. También se puede realizar la PDO para evaluar la respuesta al tratamiento; sin embargo, para tal fin recomendamos la caminata de 6 minutos $(\mathrm{C} 6 \mathrm{M})^{16}$ o la prueba de ejercicio cardiopulmonar (PECP). ${ }^{17}$ La PDO no se recomienda en pacientes que presentan hipoxemia en reposo debido a que la prueba tiene como objetivo documentar si el ejercicio submáximo induce o no desaturación de oxígeno; resulta entonces innecesario realizar la prueba en quien se encuentra hipoxémico en reposo.

Existen varios protocolos de ejercicio que pueden ser utilizados durante la PDO y la PTOS. ${ }^{6}$ Lo usual, y considerado como un estándar en este documento, es que el ejercicio realizado durante la PDO sea submáximo; es decir, que el sujeto realice un esfuerzo que en magnitud semeje al requerido para llevar a cabo las actividades de la vida diaria. En este manuscrito serán considerados únicamente los protocolos de ejercicio llevados a cabo mediante caminata en banda sin fin o caminata en corredor. ${ }^{6}$ La decisión del protocolo de ejercicio descansará sobre la disponibilidad de equipo y del estado físico del paciente.

El prototipo de las pruebas de ejercicio máximo es la PECP, la cual se lleva a cabo con un protocolo incremental (cargas de trabajo progresivamente mayores) y por lo tanto refleja la máxima capacidad que tiene un individuo para realizar ejercicio. ${ }^{17}$ Dicha prueba, aunque puede ser utilizada para evaluar desaturación de oxígeno, provee 


\section{DIFERENCIAS ENTRE LA CAMINATA DE 6 MINUTOS Y LA PDO UTILIZANDO LA CAMINATA EN CORREDOR CON DURACIÓN DE 6 MINUTOS.}

el ejercicio, cuyo análisis tiene objetivos que van más allá de la mera evaluación de la desaturación. ${ }^{7,18}$ Por ello, no se recomienda un protocolo de ejercicio incremental en una PDO. La PECP tiene sus propias indicaciones que pueden ser revisadas en documentos publicados recientemente. $^{7}$

Las recomendaciones acerca de cómo se debe de realizar la PDO en banda sin fin o mediante la caminata en corredor se encuentran en el Anexo 3 de este documento. Los criterios para considerar la PDO como «positiva» son arbitrarios y podrían ser diferentes en relación a la altitud sobre el nivel del mar. ${ }^{19}$ Proponemos criterios que consideramos «sensibles» debido a que los «falsos positivos» pueden ser sometidos a pruebas con mayor «especificidad» tal como la prueba de ejercicio cardiopulmonar con protocolo incremental que es considerada el estándar de referencia. Proponemos que la PDO (llevada a cabo en banda sin fin o caminata en corredor) sea considerada como positiva cuando en el transcurso de 6 minutos se presente cualquiera de los siguientes:

a. $\mathrm{SpO}_{2} \leq 88 \%$ de manera sostenida por al menos un minuto.

b. $\mathrm{SpO}_{2}<85 \%$ por 15 segundos

c. Disminución de al menos $4 \%$ de la $\mathrm{SpO}_{2}$ basal por al menos 1 minuto (aunque la $\mathrm{SpO}_{2}$ no esté por debajo de $88 \%$ ).

Cuando la PDO se realiza en banda sin fin y por lo tanto se dispone de un catéter arterial (Anexo 3), se incluirá también el criterio gasométrico de:

a. Disminución de $\mathrm{PaO}_{2} \geq 5 \mathrm{mmHg}$ con respecto al valor basal $y / 0$,

b. Disminución de $\mathrm{SaO}_{2} \geq 3 \%$ con respecto al valor basal.

c. $\mathrm{PaO}_{2}$ al final de la prueba $\leq 55 \mathrm{mmHg}$.

d. $\mathrm{SaO}_{2}$ al final de la prueba $\leq 88 \%$.

Algunos pacientes pueden reunir criterios de «prueba positiva» de acuerdo a los parámetros gasométricos y ser negativa al considerar la $\mathrm{SpO}_{2}$ (criterio de oximetría). En esos casos se recomienda dar preferencia a los valores obtenidos de la gasometría, dado que es el estándar de referencia. Todos los días, e idealmente antes de cada prueba, se debe de verificar la exactitud del oxímetro de pulso comparando los valores de $\mathrm{SpO}_{2}$ con los obtenidos en el analizador de gases $\left(\mathrm{SaO}_{2}\right) \cdot{ }^{20,21}$ Estos valores no deben diferir en más de 3\%. Las especificaciones técnicas de la banda sin fin, así como de los oxímetros de pulso y de la colocación del catéter arterial pueden ser consultadas en otros documentos. ${ }^{6}$
La C6M es una prueba de ejercicio submáximo que, en general, se utiliza como marcador pronóstico en enfermedades pulmonares; $;^{15,22,23}$ sirve para evaluar el efecto que tienen los tratamientos o los programas de rehabilitación sobre la capacidad física del individuo y sobre la percepción de disnea. ${ }^{24} \mathrm{El}$ objetivo primario de dicha prueba es medir la cantidad de metros que el individuo puede caminar en 6 minutos; además, es una prueba sólidamente estandarizada. ${ }^{16}$ Por ejemplo, los pacientes con enfermedad pulmonar obstructiva crónica tienen peor pronóstico de acuerdo a cuántos metros se desplazan en 6 minutos; aquellos que caminan menos de 150 metros tienen el peor pronóstico. ${ }^{22}$ La utilidad de la C6M en hipertensión arterial pulmonar también ha sido demostrada al igual que en otras enfermedades ${ }^{15}$ y se acepta que la C6M es una prueba clínicamente útil, sencilla y que requiere de mínima capacitación al personal técnico. Todo esto hace que la C6M sea una prueba de bajo costo, pudiéndose realizar prácticamente en cualquier lugar que cuente con un pasillo de 30 metros de longitud. En la actualidad la utilidad de la C6M es materia de extensa investigación en múltiples enfermedades y dadas sus bondades, será solicitada y realizada con mayor frecuencia en los laboratorios de función pulmonar.

Además de los metros caminados, la C6M permite evaluar la percepción de disnea así como la $\mathrm{SpO}_{2}$ y la frecuencia cardiaca. Debido a que usualmente, pero no de manera rutinaria, se mide la $\mathrm{SpO}_{2}$ durante la $\mathrm{C} 6 \mathrm{M}$, dicha prueba podría servir como una PDO; no obstante, la $\mathrm{C} 6 \mathrm{M}$ es una prueba diferente que, al igual que la PECP, provee información adicional a la desaturación de oxígeno. Por ello es que, cuando sólo se requiere evaluar si el paciente presenta desaturación de oxígeno al realizar ejercicio submáximo, nosotros sugerimos que se realice una versión modificada de la C6M (VMC6M). La manera de realizar la VMC6M se explica en el Anexo 3. Brevemente, a diferencia de la C6M, en la VMC6M no son considerados los metros que se desplaza el sujeto; esta prueba debe ser interrumpida cuando se han reunido ciertos criterios relacionados al estado de oxigenación (Anexo 3). Si después de 6 minutos, no se cumplen dichos criterios, se considerará a la prueba como «negativa». Debido a lo simple que es la realización de la C6M y lo útil de la información que provee, es esperable que ésta vaya sustituyendo a la VMC6M. Para los lectores interesados en revisar en detalle la C6M se sugiere el último documento de estandarización publicado por la ATS. ${ }^{8}$ 


\section{EVALUANDO EL ESTADO FÍSICO DEL PACIENTE ANTES DE LAS PRUEBAS}

Un aspecto que debe de ser evaluado antes de la realización de la PDO y de la PTOS es el estado físico del paciente. Aquellos pacientes con limitaciones físicas mayores (LFM) no podrán realizar las pruebas y cualquier evaluación funcional deberá realizarse en estado de reposo (por ejemplo, pacientes invidentes, pacientes que no puedan deambular, etc). Otro grupo de pacientes son aquellos que tienen alguna limitación física parcial (LFP); tal es el caso de pacientes que utilizan dispositivos de ayuda como «bastón», «andadera», o aquellos que utilizan prótesis de alguna extremidad pero que son capaces de desplazarse por ellos mismos. En los pacientes con LFP se puede realizar la PDO mediante la VMC6M y no recomendamos que se realice en banda sin fin. En personas sin limitaciones físicas (SLF) se recomienda que se lleve a cabo en banda sin fin de acuerdo al protocolo señalado en el Anexo 3. Cabe señalar que algunos pacientes son inicialmente considerados como SLF; sin embargo, al intentar realizar la prueba en banda sin fin, no logran adaptarse a esta modalidad o no son capaces de mantener la velocidad constante a la cual se realiza la prueba $(2.4 \mathrm{~km} / \mathrm{h})$. En dichos casos, se puede realizar la PDO o la PTOS mediante la VMC6M (Anexo 3). El estado físico del paciente puede ser evaluado por el personal técnico y en caso de duda, será necesaria la intervención del personal médico.

Una vez clasificado al paciente según su estado físico sugerimos que se sigan las recomendaciones de los algoritmos que se muestran en el Anexo 1 (Panel A, $B$ o C). Los algoritmos facilitan la toma de decisiones durante la realización de las pruebas. Antes de iniciar cualquier prueba que implique la realización de ejercicio, verifique que no existan contraindicaciones. Las contraindicaciones se pueden revisar en el Anexo 3 de este documento.

\section{PRUEBA DE TITULACIÓN DE OXÍGENO SUPLEMENTARIO}

El objetivo de la PTOS es conocer el nivel apropiado de oxígeno suplementario que el paciente necesita, ya sea en reposo o ejercicio, para mantener un umbral dado de oxigenación, por ejemplo $\mathrm{SpO}_{2} \geq 90 \%$. Existen dos formas para titular el flujo de oxígeno durante el ejercicio. ${ }^{6}$ La primera se realiza de manera continua mientras el paciente se está ejercitando; es decir, conforme el paciente camina (ya sea en la banda sin fin o en la VMC6M) se incrementa el flujo de oxígeno hasta mantener un estado estable de oxigenación. Esta forma de titulación es rápida y más cómoda para el paciente y para el técnico; sin embargo, se sobreestima el flujo de oxígeno que requiere el paciente lo cual puede ser relevante en términos de toxicidad por oxígeno y de costos generados por el dispendio de oxígeno. La segunda forma es por etapas; es decir, se hacen incrementos progresivos del flujo de oxígeno pero, a diferencia de la primera, cuando se presenta desaturación de oxígeno se le pide al paciente que se detenga, se aumenta el flujo de oxígeno y luego se reanuda el ejercicio. Esta forma, aunque consume más tiempo, permite una titulación de oxígeno de mayor exactitud y es la que recomendamos en este documento (ver en Anexo 3 el protocolo detallado de titulación de oxígeno). En el Anexo 4 reproducimos la hoja de trabajo que el personal técnico utiliza al realizar las PD y la PTOS.

\section{CONCLUSIONES}

La PD y la PTOS son realizadas con frecuencia en los laboratorios de función pulmonar y son de utilidad diagnóstica, pronóstica y terapéutica. Creemos que las recomendaciones que hemos descrito contribuyen a mejorar el proceso de estandarización de dichas pruebas y facilitan la toma de decisiones mediante la implementación de diagramas de flujo. La utilidad práctica de dichos diagramas la hemos confirmado durante las actividades cotidianas de nuestro laboratorio; sin embargo, su aplicabilidad en otros laboratorios de función pulmonar debe de ser explorada.

\section{AGRADECIMIENTOS}

Los autores desean expresar su agradecimiento a Óscar Robles Lazcano, Adela Durán Cuéllar, Rosa María Talavera Corona, Isabel Salas Escamilla y Anaid Bautista Bernal por su invaluable soporte técnico.

\section{REFERENCIAS}

1. Miller MR, Hankinson J, Brusasco V, et al. Standardization of spirometry. Eur Respir J 2005; 26: 319-338.

2. Miller MR, Crapo R, Hankinson J, et al. General considerations for lung function testing. Eur Respir J 2005; 26: 153-161.

3. Wanger J, Clausen JL, Coates A, et al. Standardization of the measurement of lung volumes. Eur Respir J 2005; 26: 511-522.

4. Macintyre N, Crapo RO, Viegi G, et al. Standardization of the singlebreath determination of carbon monoxide uptake in the lung. Eur Respir J 2005; 26: 720-735.

5. Pellegrino R, Viegi G, Brusasco V, et al. Interpretative strategies for lung function tests. Eur Respir J 2005; 26: 948-968.

6. Wanger J CR, Irvin C. Pulmonary Function Laboratory Management and Procedure Manual. A project of the American Thoracic Society. Pulse Oximetry with Exercise: Assessment of desaturation, oxygen titration and distance walked. American Thoracic Society, American Lung Association. Chapter 17. 2000. 
7. Palange P, Ward SA, Carlsen $\mathrm{KH}$, et al. Recommendations on the use of exercise testing in clinical practice. Eur Respir J 2007; 29: 185-209.

8. Casanova C, Cote C, Marin JM, et al. Distance and oxygen desaturation during the 6-min walk test as predictors of long-term mortality in patients with COPD. Chest 2008; 134: 746-752.

9. Sandland CJ, Morgan MD, Singh SJ. Detecting oxygen desaturation in patients with COPD: incremental versus endurance shuttle walking. Respir Med 2008; 102: 1148-1152

10. Garcia-Talavera I, Garcia CH, Macario CC, et al. Time to desaturation in the 6-min walking distance test predicts 24-hour oximetry in COPD patients with a $\mathrm{PO}_{2}$ between 60 and $70 \mathrm{mmHg}$. Respir Med 2008; 102: 1026-1032.

11. Takigawa N, Tada A, Soda R, et al. Distance and oxygen desaturation in 6-min walk test predict prognosis in COPD patients. Respir Med 2007; 101: 561-567.

12. Christensen CC, Ryg MS, Edvardsen A, et al. Relationship between exercise desaturation and pulmonary haemodynamics in COPD patients. Eur Respir J 2004; 24: 580-586.

13. Brunelli $\mathrm{A}, \mathrm{Al}$ Refai $\mathrm{M}$, Monteverde $\mathrm{M}$, et al. Predictors of exercise oxygen desaturation following major lung resection. Eur J Cardiothorac Surg 2003; 24: 145-148.

14. Hadeli KO, Siegel EM, Sherrill DL, et al. Predictors of oxygen desaturation during submaximal exercise in 8,000 patients. Chest 2001; 120: 88-92.

15. Paciocco G, Martinez FJ, Bossone E, et al. Oxygen desaturation on the six-minute walk test and mortality in untreated primary pulmonary hypertension. Eur Respir J 2001; 17: 647-652.
16. ATS statement: guidelines for the six-minute walk test. Am J Respir Crit Care Med 2002; 166: 111-117.

17. ATS/ACCP Statement on cardiopulmonary exercise testing. Am J Respir Crit Care Med 2003; 167: 211-277.

18. Poulain $M$, Durand F, Palomba B, et al. 6-minute walk testing is more sensitive than maximal incremental cycle testing for detecting oxygen desaturation in patients with COPD. Chest 2003; 123: 1401-1407.

19. Perez Padilla JR, Vazquez Garcia JC. [Estimation of gasometric values at different altitudes above sea level in Mexico]. Rev Invest Clin 2000; 52: 148-155.

20. Torre-Bouscoulet L, Chavez-Plascencia E, Vazquez-Garcia JC, et al. [Precision and accuracy of "a pocket" pulse oximeter in Mexico City]. Rev Invest Clin 2006; 58: 28-33.

21. Escourrou PJ, Delaperche MF, Visseaux A. Reliability of pulse oximetry during exercise in pulmonary patients. Chest 1990; 97: 635-638.

22. Celli BR, Cote CG, Marin JM, et al. The body-mass index, airflow obstruction, dyspnea, and exercise capacity index in chronic obstructive pulmonary disease. N Engl J Med 2004; 350: 1005-1012.

23. Reesink HJ, van der Plas MN, Verhey NE, et al. Six-minute walk distance as parameter of functional outcome after pulmonary endarterectomy for chronic thromboembolic pulmonary hypertension. J Thorac Cardiovasc Surg 2007; 133: 510-516.

24. Verrill D, Barton C, Beasley W, et al. The effects of short-term and long-term pulmonary rehabilitation on functional capacity, perceived dyspnea, and quality of life. Chest 2005; 128: 673-683.

25. Borg GA. Psychophysical bases of perceived exertion. Med Sci Sports Exerc 1982; 14: 377-381. 


\section{Anexo 1: Panel A}

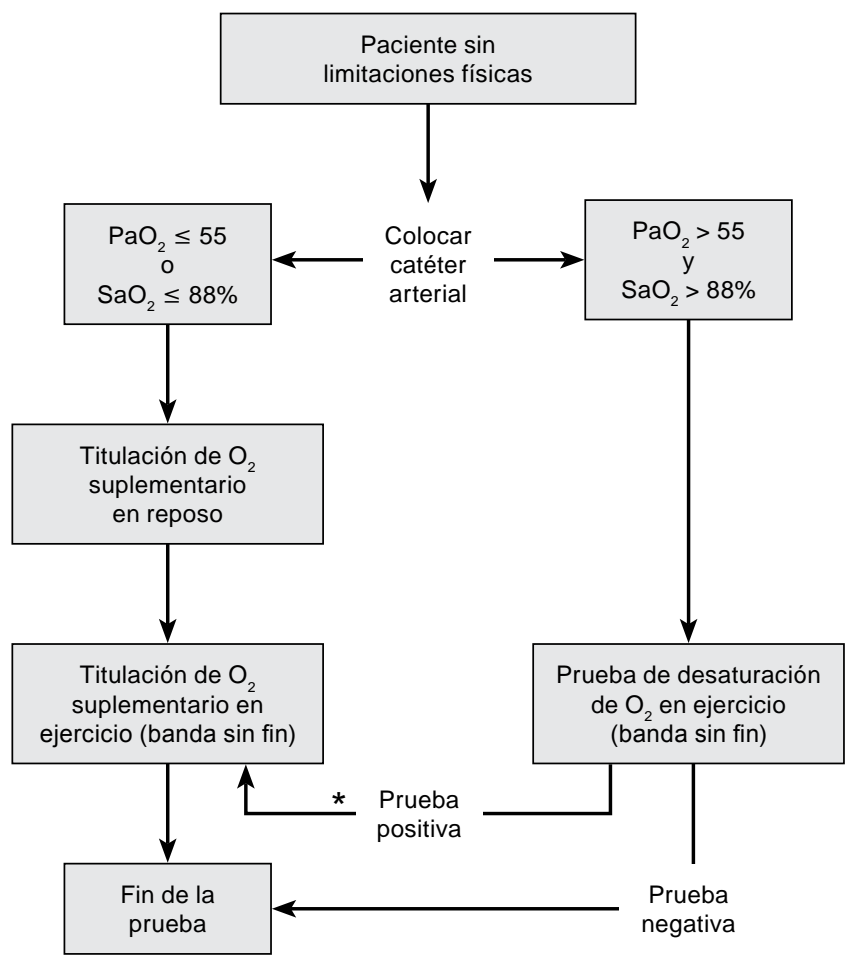

* La titulación de $\mathrm{O}_{2}$ suplementario en ejercicio (banda sinfín) se realiza siempre y cuando la prueba de desaturación haya sido positiva por el criterio de $\mathrm{SaO}_{2}<88 \%$ o $\mathrm{PaO}_{2}<55 \mathrm{mmHg}$.

\section{Anexo 1: Panel B}

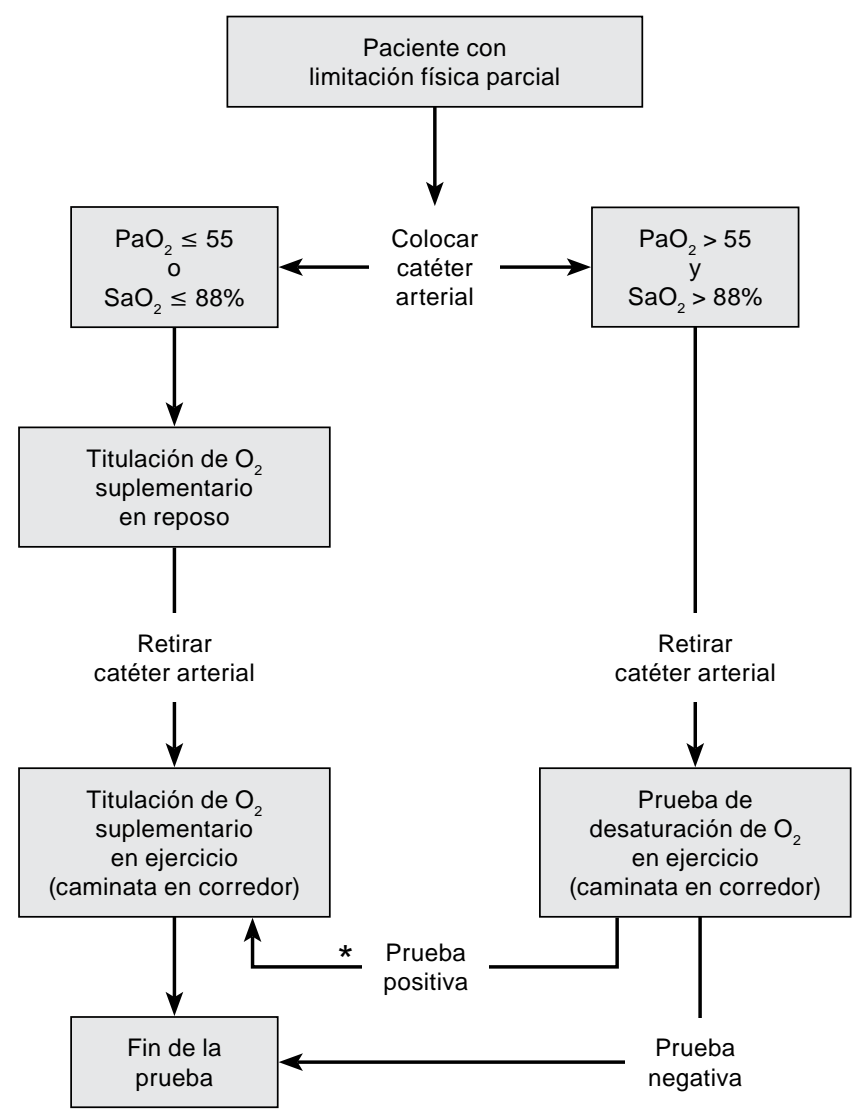

* La titulación de $\mathrm{O}_{2}$ suplementario en ejercicio (caminata en corredor) se realiza siempre y cuando la prueba de desaturación haya sido positiva por el criterio de $\mathrm{SpO}_{2}<88 \%$.

\section{Anexo 1: Panel C}

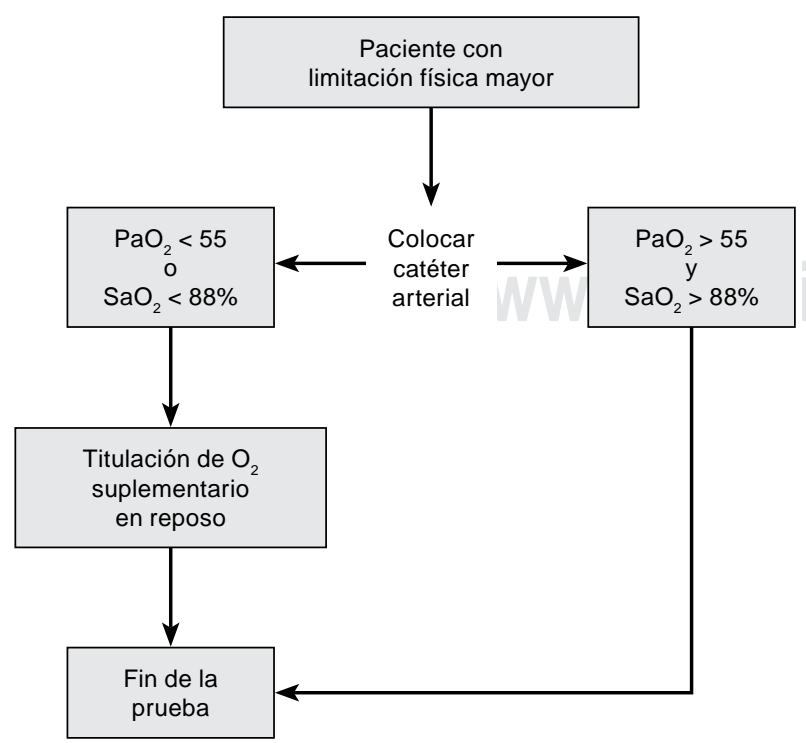

\section{Anexo 2}

Escala de Borg para disnea y fatiga. ${ }^{25}$

0 Ninguna

0.5 Muy, muy leve (apenas perceptible)

1 Muy leve

2 Leve

3 Moderada

4 Algo intensa

5 Intensa

6

7 Muy intensa

8

9 Muy, muy intensa (casi máxima)

10 Máxima 


\section{Anexo 3}

\section{Realización de la prueba de desaturación y titulación de oxígeno suplementario en reposo y ejercicio}

1. Evalúe si existen contraindicaciones para la realización de cualquiera de las pruebas que se realizan durante el ejercicio. Las contraindicaciones son:

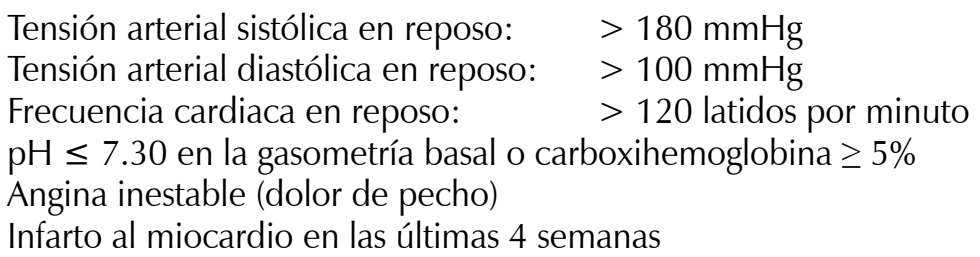

Si el paciente presenta una o más contraindicaciones, informe de inmediato al personal médico del laboratorio.

2. Clasifique al paciente de acuerdo al estado funcional en: (la clasificación del estado funcional le permitirá conocer el algoritmo que debe de seguir de acuerdo al Anexo 1)

- Sin limitaciones físicas (SLF): Sujeto que no presenta impedimentos físicos para realizar la(s) prueba(s) en banda sin fin manteniendo una velocidad constante de $2.4 \mathrm{~km} / \mathrm{h}$.

- Limitación física parcial (LFP): Paciente que requiere auxiliares ortopédicos (uso de bastón, andadera, prótesis). También se considerará LFP a aquel paciente que no sea capaz de mantener la velocidad constante de $2.4 \mathrm{~km} / \mathrm{h}$ en la banda sin fin. Los pacientes con LFP deberán ser evaluados únicamente mediante pruebas de caminata en corredor.

- Limitación física mayor (LFM): Es aquella que impide la correcta realización de la prueba, ya sea en banda sin fin o en caminata en corredor (ej: paciente invidente, sordo, ausencia de una extremidad, etc.). A los pacientes con LFM sólo se les realizarán pruebas en reposo (ej: Gasometría arterial en reposo, titulación de oxígeno suplementario en reposo).

\section{Prueba para valorar desaturación de oxígeno durante el ejercicio (en banda sin fin)}

La prueba consiste en que el paciente camine sobre una banda sin fin a una velocidad constante de $2.4 \mathrm{~km} / \mathrm{h}$ durante un máximo de 6 minutos.

La ejecución de la prueba es de la siguiente manera:

1. Preséntese con el paciente o su familiar y revise la solicitud.

2. Verifique la identidad del paciente.

3. Explique la prueba al paciente y/o su familiar y obtenga la autorización por escrito.

4. Después de que el paciente haya permanecido en reposo y en posición sedente durante 5 minutos, obtenga y registre la puntuación en la escala de disnea y fatiga de Borg y mida y registre la $\mathrm{SpO}_{2}$ así como la frecuencia cardiaca y la presión arterial.

5. Verifique que no existan contraindicaciones (ver arriba).

6. Una vez realizada la prueba de Allen, coloque un catéter en la arteria radial de la mano no dominante.

7. Espere 5 minutos y realice una gasometría arterial. Registre los valores gasométricos.

8. Solicite al paciente que suba a la banda.

9. Se inicia la prueba a velocidad constante de $2.4 \mathrm{~km} / \mathrm{h}$ con duración máxima de 6 minutos

10. La prueba se deberá interrumpir si se presenta alguno de los siguientes:

a. $\mathrm{SpO}_{2} \leq 88 \%$ de manera sostenida por al menos un minuto.

b. $\mathrm{SpO}_{2}<85 \%$ por 15 segundos o

c. Disminución de al menos $4 \%$ de la $\mathrm{SpO}_{2}$ basal por al menos 1 minuto (aunque no esté por debajo de 88\%).

d. El paciente lo solicita (especificar el motivo; $p$ ej: náusea, dolor en el pecho, calambres).

e. El paciente no logra caminar a la velocidad constante de $2.4 \mathrm{~km} / \mathrm{h}$. 
11. En caso de no presentar alguno de los anteriores (incisos «a», «b» $O$ «C») la prueba se concluye al completar 6 minutos y esto define a la prueba como NEGATIVA.

12. En cualquiera de los casos, realice una gasometría arterial en los últimos 15 segundos antes de finalizar la prueba y registre los valores.

13. Al concluir la prueba y aún el paciente sobre la banda, obtenga y registre la puntuación en la escala de disnea y fatiga de Borg y mida y registre la $\mathrm{SpO}_{2}$, la frecuencia cardiaca y la presión arterial.

14. Solicite al paciente que se siente, retire el catéter arterial e informe al paciente que la prueba ha concluido.

15. Si el paciente manifiesta algún malestar notifíquelo de inmediato al personal médico.

16. Si durante la prueba el paciente presenta cualquiera de los criterios mencionados en los incisos «a», «b» $O$ «C»; la prueba se considera POSITIVA.

17. Si la prueba fue interrumpida antes de transcurrir 6 minutos porque el paciente así lo solicitó (usualmente debido a la aparición de síntomas), informe de inmediato al personal médico.

18. Si la prueba fue interrumpida antes de transcurrir 6 minutos porque el paciente no logró caminar a la velocidad constante de $2.4 \mathrm{~km} / \mathrm{h}$; anótelo en la hoja técnica correspondiente y realice la prueba mediante la caminata en corredor como a continuación se explica.

\section{Prueba para valorar desaturación de oxígeno durante el ejercicio (versión modificada de la prueba de caminata de 6 minutos).}

Consiste en caminar en un pasillo de 30 metros durante un máximo de 6 minutos siguiendo las recomendaciones técnicas sugeridas por la ATS en 2002. ${ }^{16}$

La ejecución de la prueba es de la siguiente manera:

1. Preséntese con el paciente o su familiar y revise la solicitud.

2. Verifique la identidad del paciente.

3. Explique la prueba al paciente y/o su familiar y obtenga la autorización por escrito.

4. Después de que el paciente haya permanecido en reposo y en posición sedente durante 5 minutos, obtenga y registre la puntuación en la escala de disnea y fatiga de Borg y mida y registre la $\mathrm{SpO}_{2}$ así como la frecuencia cardiaca y la presión arterial.

5. Verifique que no existan contraindicaciones.

6. Una vez realizada la prueba de Allen, realice una gasometría arterial por punción en la arterial radial de la mano no dominante.

7. Inicie la prueba de caminata en corredor siguiendo las recomendaciones técnicas de la ATS. ${ }^{16}$

8. La prueba se deberá interrumpir si se presenta alguno de los siguientes:

a. $\mathrm{SpO}_{2} \leq 88 \%$ de manera sostenida por al menos un minuto.

b. $\mathrm{SpO}_{2}<85 \%$ por 15 segundos o

c. Disminución de al menos $4 \%$ de la $\mathrm{SpO}_{2}$ basal por al menos 1 minuto (aunque no esté por debajo de 88\%)

d. El paciente lo solicita (especificar el motivo).

9. En caso de no presentar alguno de los anteriores (incisos «a», «b» $\mathrm{o}$ «C») la prueba se concluye al completar 6 minutos de caminata y esto define a la prueba como NEGATIVA.

10. Al concluir la prueba solicite al paciente que se siente, obtenga y registre la puntuación en la escala de disnea y fatiga de Borg y mida y registre la $\mathrm{SpO}_{2}$, la frecuencia cardiaca y la presión arterial.

11. Informe al paciente que la prueba ha concluido.

12. Si el paciente manifiesta algún malestar notifíquelo de inmediato al personal médico.

13. Si durante la prueba el paciente presenta cualquiera de los incisos «a», «b» o «c»; la prueba se considera POSITIVA.

14. Si la prueba fue interrumpida antes de transcurrir 6 minutos porque el paciente así lo solicitó (usualmente debido a la aparición de síntomas), informe de inmediato al personal médico. 


\section{Titulación de oxígeno suplementario en reposo}

La ejecución de la prueba es de la siguiente manera:

1. Preséntese con el paciente o su familiar y revise la solicitud.

2. Verifique la identidad del paciente.

3. Explique la prueba al paciente y/o su familiar y obtenga la autorización por escrito.

4. Solicite al paciente que permanezca sentado por un periodo de 5 minutos.

5. Una vez realizada la prueba de Allen, coloque un catéter en la arteria radial de la mano no dominante.

6. Espere 5 minutos y realice una gasometría arterial. Registre los valores gasométricos.

7. Inicie la titulación de oxígeno suplementario a un flujo de $1 \mathrm{~L} / \mathrm{min}$ por puntas nasales.

8. Incrementar el flujo de oxígeno $1 \mathrm{~L} /$ min cada 5 minutos, hasta alcanzar una $\mathrm{SpO}_{2}$ mínima estable (5 minutos) de $92 \%$.

9. Una vez alcanzada la $\mathrm{SpO}_{2}$ de al menos $92 \%$, se deberá realizar una segunda gasometría. Registre los valores gasométricos.

10. Informe al paciente que la prueba ha concluido y retire el catéter arterial.

11. Si el paciente manifiesta algún malestar notifíquelo de inmediato al personal médico

\section{Titulación de oxígeno suplementario en ejercicio (banda sin fin)}

1. Preséntese con el paciente o su familiar y revise la solicitud.

2. Verifique la identidad del paciente.

3. Explique la prueba al paciente y/o su familiar y obtenga la autorización por escrito.

4. Después de mantenerse en reposo y en posición sedente durante 5 minutos, mida y registre la $\mathrm{SpO}_{2}$ así como la frecuencia cardiaca y la presión arterial.

5. Verifique que no existan contraindicaciones.

6. Una vez realizada la prueba de Allen, coloque un catéter en la arteria radial de la mano no dominante.

7. Espere 5 minutos y realice una gasometría arterial. Registre los valores gasométricos.

8. Solicite al paciente que suba a la banda.

9. Se inicia la prueba a velocidad constante de $2.4 \mathrm{~km} / \mathrm{h}$ administrando al paciente 1 litro de oxígeno por arriba del flujo requerido en la titulación en reposo. Si en reposo no requiere oxígeno suplementario pero se documentó una prueba de desaturación de oxígeno POSITIVA por los criterios «a» o «b» (NO por el criterio del inciso «C»), se inicia la titulación en ejercicio a un litro por minuto.

10. Si en el transcurso de la prueba presenta:

a. $\mathrm{SpO}_{2} \leq 88 \%$ por lo menos un minuto o

b. $\mathrm{SpO}_{2} \leq 85 \%$ por lo menos 15 segundos

interrumpir la prueba y solicitar al paciente que se siente.

11. Aumentar 1 litro el flujo de oxígeno y esperar 5 minutos.

12. Al término de este periodo, solicitar al paciente que suba nuevamente a la banda y reinicie la prueba a una velocidad de $2.4 \mathrm{~km} / \mathrm{h}$. En caso de que nuevamente presente desaturación (ver arriba número 10), interrumpa la prueba y repita el procedimiento (ver arriba números 11 y 12).

13. La prueba se concluye cuando:

- Han transcurrido 6 minutos caminando a velocidad constante sin presentar desaturación (ver arriba número 10).

- El flujo máximo de oxígeno suplementario es de 6 L/min.

- El paciente lo solicita.

14. Realice una gasometría arterial en los últimos 15 segundos antes de finalizar la prueba y registre los valores.

15. Al concluir la prueba mida y registre la $\mathrm{SpO}_{2}$ así como la frecuencia cardiaca y la presión arterial.

16. Solicite al paciente que se siente, retire el catéter arterial e informe al paciente que la prueba ha concluido. 
17. Si el paciente manifiesta algún malestar notifíquelo de inmediato al personal médico.

18. El flujo de oxígeno durante el ejercicio (flujo titulado de oxígeno) corresponde a aquel que le permitió al paciente caminar a velocidad constante $(2.4 \mathrm{~km} / \mathrm{h})$ durante 6 minutos sin presentar desaturación.

\section{Titulación de oxígeno suplementario en ejercicio (caminata en corredor; versión modificada de la prueba de caminata de 6 minutos)}

1. Verifique la identidad del paciente.

2. Preséntese con el paciente o su familiar y revise la solicitud.

3. Explique la prueba al paciente y/o su familiar y obtenga la autorización por escrito.

4. Después de mantenerse en reposo y en posición sedente durante 5 minutos, mida y registre la $\mathrm{SpO}_{2}$ así como la frecuencia cardiaca y la presión arterial.

5. Verifique que no existan contraindicaciones.

6. Inicie la prueba bajo las recomendaciones internacionales de la ATS $^{6}$ administrando al paciente 1 litro de oxígeno por arriba del flujo requerido en la titulación en reposo. Si en reposo no requiere oxígeno suplementario pero se documentó una prueba de desaturación de oxígeno POSITIVA por los criterios «a» o «b» (NO por el criterio del inciso «८), se inicia la titulación en ejercicio a 1 litro por minuto.

7. Si en el transcurso de la prueba presenta:

a. $\mathrm{SpO}_{2} \leq 88 \%$ por lo menos un minuto o

b. $\mathrm{SpO}_{2} \leq 85 \%$ por lo menos 15 segundos

interrumpir la prueba y solicitar al paciente que se siente.

8. Aumentar 1 litro el flujo de oxígeno y esperar 5 minutos.

9. Al término de este periodo solicitar al paciente que reinicie la caminata. En caso de que nuevamente presente desaturación (ver arriba número 7), interrumpa la prueba y repita el procedimiento (ver arriba números 8 y 9).

10. La prueba se concluye cuando:

- Han transcurrido 6 minutos sin presentar desaturación (ver arriba número 7).

- El flujo máximo de oxígeno suplementario es de $6 \mathrm{~L} / \mathrm{min}$.

- El paciente lo solicita.

11. Al concluir la prueba mida y registre la $\mathrm{SpO}_{2}$ así como la frecuencia cardiaca y la presión arterial.

12. Solicite al paciente que se siente e infórmele que la prueba ha concluido.

13. Si el paciente manifiesta algún malestar notifíquelo de inmediato al personal médico.

14. El flujo de oxígeno durante el ejercicio (flujo titulado de oxígeno) corresponde a aquel que le permitió al paciente caminar durante 6 minutos sin presentar desaturación.

\section{Prueba de Allen}

Es una prueba sencilla que se utiliza para verificar la viabilidad de la circulación colateral de la mano que se va a puncionar para obtener la muestra de sangre arterial (arteria radial). Se realiza de la siguiente manera:

1. Localice el pulso radial y cubital de la mano que se planea puncionar y oprímalos firmemente de manera simultánea.

2. Pida al paciente que abra y cierre el puño vigorosamente hasta que aparezca una coloración pálida en la palma, lo que indica isquemia distal.

3. Con la mano del paciente extendida, libere la compresión de la región cubital.

4. Registre el tiempo en que reaparece la coloración palmar habitual.

Se considera una circulación colateral adecuada cuando la coloración normal aparece antes de 15 segundos. 


\section{Anexo 4}

\section{Prueba de Desaturación de Oxígeno}

Nombre:

Fecha:

Género:
Edad:

Técnico:
Expediente:

Diagnóstico:

Sin limitaciones físicas

Limitación física parcial

Limitación física mayor (sólo realizar evaluación en reposo).

\begin{tabular}{|l|l|l|l|l|l|l|l|l|l|l|}
\hline Tiempo & $\mathrm{SpO}_{2}$ & $\mathrm{FC}$ & $\mathrm{TA}$ & $\mathrm{pH}^{*}$ & $\mathrm{PaCO}_{2}$ & $\mathrm{PaO}_{2}$ & $\mathrm{SaO}_{2}$ & Disnea & Fatiga & Observaciones \\
\hline Reposo & & & & & & & & & & \\
\hline Minuto 1 & & & & & & & & & & \\
\hline Minuto 2 & & & & & & & & & & \\
\hline Minuto 3 & & & & & & & & & & \\
\hline Minuto 4 & & & & & & & & & & \\
\hline Minuto 5 & & & & & & & & & & \\
\hline Minuto 6 & & & & & & & & & & \\
\hline Final & & & & & & & & & & \\
\hline
\end{tabular}

${ }^{*}$ Si el pH es $<7.30$ No realizar la prueba

La prueba fue finalizada por:

Desaturación de oxígeno

$\mathrm{SpO}_{2} \leq 88 \%$ por al menos un minuto

$\mathrm{SpO}_{2}<85 \%$ por al menos 15 segundos

Disminución de al menos $4 \%$ de la $\mathrm{SpO}_{2}$ basal por al menos un minuto

Completó 6 minutos sin presentar desaturación.

El paciente lo solicitó (especificar motivo en observaciones).

Paciente NO pudo caminar a 2.4 km/h (retirar la línea arterial y realizar la prueba de desaturación en caminata de 6 minutos).

\section{Prueba de Titulación de Oxígeno}

\begin{tabular}{|l|l|l|l|l|l|l|l|l|l|}
\hline Tiempo (minutos) & $\mathrm{SpO}_{2}$ & $\mathrm{FC}$ & $\mathrm{TA}$ & $\mathrm{pH}$ & $\mathrm{PaCO}_{2}$ & $\mathrm{PaO}_{2}$ & $\mathrm{SaO}_{2}$ & $\mathrm{O}_{2} \mathrm{~L} / \mathrm{min}$ & Observaciones \\
\hline Reposo & & & & & & & & & \\
\hline Titulación en reposo & & & & & & & & & \\
\hline Etapa 1 & & & & & & & & & \\
\hline Etapa 2 & & & & & & & & & \\
\hline Etapa 3 & & & & & & & & & \\
\hline Etapa 4 & & & & & & & & & \\
\hline Etapa 5 & & & & & & & & & \\
\hline Etapa 6 & & & & & & & & & \\
\hline Final
\end{tabular}

Nota 1: La gasometría basal EN REPOSO es válida para las dos pruebas. Cuando sean solicitadas las dos pruebas el máximo de gasometría a realizar serán 3.

Nota 2: La duración máxima de cada etapa de la prueba de titulación de oxígeno será de 6 minutos.

Nota 3: Si la titulación se hace en caminata no realizar la gasometría final.

Nota 4: No haga anotaciones en los espacios sombreados. 